Sādhanā Vol. 39, Part 2, April 2014, pp. 467-485. (C) Indian Academy of Sciences

\title{
Uncertainty modelling of atmospheric dispersion by stochastic response surface method under aleatory and epistemic uncertainties
}

\author{
RITUPARNA CHUTIA $^{1, *}$, SUPAHI MAHANTA ${ }^{2}$ and D DATTA ${ }^{3}$ \\ ${ }^{1}$ Department of Mathematics, Gauhati University, Guwahati, Assam 781 014, India \\ ${ }^{2}$ Department of Statistics, Gauhati University, Guwahati, Assam 781 014, India \\ ${ }^{3}$ Health Physics Division, Bhabha Atomic Research Centre, Mumbai 400 085, India \\ e-mail: rituparnachutia7@gmail.com
}

MS received 15 January 2013; revised 14 October 2013; accepted 26 October 2013

\begin{abstract}
The parameters associated to a environmental dispersion model may include different kinds of variability, imprecision and uncertainty. More often, it is seen that available information is interpreted in probabilistic sense. Probability theory is a well-established theory to measure such kind of variability. However, not all available information, data or model parameters affected by variability, imprecision and uncertainty, can be handled by traditional probability theory. Uncertainty or imprecision may occur due to incomplete information or data, measurement error or data obtained from expert judgement or subjective interpretation of available data or information. Thus for model parameters, data may be affected by subjective uncertainty. Traditional probability theory is inappropriate to represent subjective uncertainty. Possibility theory is used as a tool to describe parameters with insufficient knowledge. Based on the polynomial chaos expansion, stochastic response surface method has been utilized in this article for the uncertainty propagation of atmospheric dispersion model under consideration of both probabilistic and possibility information. The proposed method has been demonstrated through a hypothetical case study of atmospheric dispersion.
\end{abstract}

Keywords. Uncertainty; polynomial chaos expansion; fuzzy set theory; cumulative distribution function; uniform distribution; membership function.

\section{Introduction}

Atmospheric dispersion models are mathematical expressions relating the emission of material into the atmosphere to the downwind ambient concentration of the material. The main aim of

*For correspondence 
dispersion modelling is to estimate the concentration of a pollutant at a particular receptor point by calculating the basic information about the source of the pollutant and the meteorological conditions. Concentration of an air pollutant at a given place is a function of a large number of variables such as rate of emission, distance of the receptor from the source and atmospheric conditions. The important atmospheric conditions are wind speed, wind direction and vertical temperature structure of local atmosphere. Air pollution dispersion models are subject to scientific uncertainty, but the way this is handled, for air quality management policy differs depending on the scale of modelling and the impact under consideration (Colvile et al 2002). Information about parameters in dispersion models can be gained through measurement, calibration, expert judgement, etc. However, value of these parameters may be subject to uncertainty due to lack of measurement point and over-calibration or inaccurate expert judgement. Inherent uncertainty of the input parameters is one of the main cause of uncertainty in model output. Parameter uncertainty is present because a single value of a parameter cannot completely characterize a modelling platform (Saeedi et al 2008).

A large number of mathematical methods have been developed for handling those uncertainties. Probabilistic and statistical methods available are usually preferred for analysis of aleatory uncertainty. Some of the methods for uncertainty propagation are available in Du \& Chen (2001), Hessian et al (1996), Hoybe (1998), Isukapalli \& Georgopoulos (1998), Mahadevan \& Raghothamachar (2000), Wang (1999). The most popular existing method of aleatory uncertainty analysis is Monte-Carlo Simulation (Mahadevan \& Raghothamachar 2000) where large number of parameters are generated based on the distribution functions of the parameters and propagated to draw distribution function for model output. Most important methods of handling epistemic uncertainties consist of interval analysis (Sengupta \& Pal 2000), possibility theory (Dubois \& Parde 1988) and fuzzy set theory (Zadeh 1978). On the other hand, several competing approaches have been suggested when both aleatory and epistemic uncertainties are present (Baudrit et al 2007; Ferson et al 1999; Guyonnet et al 2003; Kentel \& Aral 2005).

With advances in fuzzy mathematics, researchers have applied this theory into different fields. Some of the literature describing the application of fuzzy mathematics and fuzzy logic in the field of atmospheric dispersion are Fakhraee et al (2007) and Saeedi et al (2008), where the authors used direct fuzzy modelling to construct fuzzy-rule-base considering the Gaussian plume function. An approach to non-probabilistic sensitivity analysis of dispersion model RIMPUFF using the Hartley-like measure is explained explicitly by Chutia et al (2013). Also, a non-probabilistic sensitivity and uncertainty of atmospheric dispersion using fuzzy set theory can be found in Chutia et al (2013). Further, a hybrid method of uncertainty analysis of atmospheric dispersion under the presence of fuzzy numbers and imprecise probability as input uncertainties is discussed by Chutia et al (2013).

The objective of this paper is to present a theory of the stochastic response surface method (SRSM) under both the probability and the possibility theories and its application through a hypothetical case study of atmospheric dispersion. The previous uncertainty modelling of atmospheric dispersion was mostly based on either stochastic or fuzzy approaches. But only a few of previous studies on atmospheric dispersion admits integration of both aleatory and epistemic uncertainties into a single modelling platform. So, an attempt has been made to combine these types of uncertainty into a single modelling platform. Also the SRSM is a purely probabilistic method where the input parameters have probability density functions. Here an attempt has been made to incorporate possibility information into it. The primary purpose is to promote a methodology for uncertainty modelling when information about model parameters are both 
probabilistic and fuzzy. The proposed method is rather an extension of the SRSM to a Hybrid SRSM. The proposed method has been successfully applied to a hypothetical atmospheric dispersion model and the results are interpreted in terms of percentile.

The structure of the paper is as follows. In section 2, general concept of different types of uncertainty and a brief concept of fuzzy set theory are laid down. Also the different uncertainty modelling method under probabilistic, possibility and hybrid (probabilistic-possibility) are discussed. In section 3, the SRSM under both the probabilistic and the possibility environment is discussed. In section 4, a hypothetical atmospheric dispersion model is considered as a case study. The concerned model and the input data are taken from Abrahamsson (2002). Finally, in section 5, the conclusions have been provided.

\section{Types of uncertainty and approaches of analysis}

\subsection{Uncertainty representation}

Two types of uncertainty exist in the real world, namely aleatory and epistemic. Uncertainty which results from the fact that a system behaves in random ways is aleatory and uncertainty that results due to lack of knowledge about fundamental phenomena, system and property of analysis performing the analysis is epistemic. If sufficient amount of information are available, stochastic simulations with parameters generated by probability density functions can be used to assess aleatory uncertainty. This type of uncertainty is also referred to as irreducible, stochastic, or random uncertainty. On the other hand, reasons for epistemic uncertainty is lack of reliable experimental data, which can be ambiguous, conflicting, insufficient, or not in agreement with existing conceptual models used for predictions and quantifying uncertainty (Faybishenko 2010).

Uncertainties can be represented in many ways. The literature describes many types of uncertainty representation. Among the most encountered uncertainty representations are probability distributions, interval, probability bounds and fuzzy numbers. Aleatory uncertainties are represented by probability distributions and fuzzy is the representation of epistemic uncertainty. Probability bounds and interval are used to represent both aleatory and epistemic uncertainties. Probability bounds are constructed from parametric probability distributions where the parameters are uncertain. Probability bounds can also be constructed without making any assumption about the shape of the distribution (Ferson et al 1999). Uncertainty may not always be due to randomness, and if available information is not much informative or subjective or in the form of expert judgement or with imprecise boundaries, fuzzy set introduced by Zadeh (1965) is the best tool to represent it.

\subsection{Fuzzy set theory}

The application of fuzzy set theory has been evident in different fields of study. The concept of fuzzy numbers arise from the experiences of everyday life when many phenomena which can be quantified but cannot be characterised in terms of absolutely precise numbers. Fuzzy numbers are convex and normalised fuzzy sets which are defined on the set of real numbers. The membership function of a fuzzy number assigns degree of 1 to the most probable value, also called the mean value and lower degrees to other numbers which reflect their proximity to the most probable value according to the used membership function. Thus, the membership function decreases from 1 to 0 on both sides of the most probable value. 
As an illustration, let $\eta=(a, b, c)$ be triangular fuzzy number defined in a real line $R$. The membership function $\mu_{\eta}(x)$ of $\eta$ is defined as

$$
\mu_{\eta}(x)= \begin{cases}\frac{x-a}{b-a}, & \text { if } a \leq x \leq b \\ \frac{c-x}{c-b}, & \text { if } b \leq x \leq c \\ 0, & \text { otherwise }\end{cases}
$$

The membership function of a trapezoidal fuzzy number $\beta=(a, b, c, d)$ is defined as

$$
\mu_{\beta}(x)= \begin{cases}\frac{x-a}{b-a}, & \text { if } a \leq x \leq b \\ 1, & \text { if } b \leq x \leq c \\ \frac{d-x}{d-c}, & \text { if } c \leq x \leq d \\ 0, & \text { otherwise }\end{cases}
$$

The most important concept of fuzzy set is $\alpha$-level set or $\alpha$-cuts. The $\alpha$-cut of a fuzzy number is a interval defined for a specific value of the membership function. The $\alpha$-cut of the triangular fuzzy number $\eta$ is defined as $[a+\alpha(b-a), c+\alpha(b-c)]$, where $\alpha=\left[0, \frac{1}{p}, \frac{2}{p}, \cdots, 1\right], p$ is any natural number and for a trapezoidal fuzzy number defined by the membership function 2 the $\alpha$-cut is defined as $[a+\alpha(b-a), d+\alpha(c-d)]$, where $\alpha=\left[0, \frac{1}{p}, \frac{2}{p}, \cdots, 1\right], p$ is any natural number. The core of a membership function for some fuzzy set is defined as that region of the universe that is characterized by complete and full membership in the set. The support of a membership function for some fuzzy set is defined as that region of the universe that is characterized by non-zero membership in the set.

\subsection{Uncertainty analysis}

Most commonly used approach for propagating uncertainty in model input parameters are the analytical and the Monte Carlo (MC) Methods. In analytical methods, (Adomian 1980; Isukapalli 1999), output uncertainties are represented explicitly as functions of input uncertainties. These methods cannot be applied to complex non-linear models with large number of uncertainties, but are useful when only small range of uncertainty is considered. Monte Carlo Methods (Frey \& Bammi 2003; Ibrahim 1998; Isukapalli 1999; Mahadevan \& Raghothamachar 2000), involve the estimation of probability density functions for selected model outputs by performing a sufficiently large number of model runs with randomly sampled inputs. Firstorder second-moment analysis (Hoybe 1998), stochastic response surface methods (Isukapalli \& Georgopoulos 1998), Polynomial Chaos Expansion Method (Wang 1999) and Reliability Analysis based approaches (Du \& Chen 2001) are some of the methods that are applicable to aleatory uncertainty analysis.

The growing trend of uncertainty analysis and uncertainty representation need studies in coupling various types of uncertainty existing in a system. Extensive studies are made by coupling probabilistic and fuzzy knowledge in a single platform. The Hybrid Method (Guyonnet et al 2003) is a method that combines aleatory and epistemic uncertainties. In fact this is a method for combining the model parameter uncertainty which combines Monte-Carlo technique with extension principle of fuzzy set theory. The Two-phase Monte-Carlo simulation approach can be used to treat both probabilistic and possibility uncertainties. The Two-phase Monte-Carlo approach allows the evaluation and propagation of natural stochastic variability and knowledge uncertainty separately (Hessian et al 1996). The probability box (p-box) approach combines intervals and probability density functions, by imposing bounds on a cumulative distribution 
function (CDF) used to express different sources of uncertainty. This method provides an envelope of distribution functions, which bounds all possible estimations (Ferson et al 1999; Ferson \& Ginzburg 1995; Tucker \& Ferson 2003). The Probabilistic-Fuzzy approach (Kentel \& Aral 2004) is also a hybrid method which combines probabilistic and fuzzy parameters and the combined utilization of fuzzy and random variables produces membership functions of model output at different fractiles of model output as well as probability distributions of model output for various $\alpha$-cut levels of the membership function. The 2D Fuzzy Monte Carlo Analysis (2D FMCA) (Kentel \& Aral 2005) is a modification of 2D Monte Carlo Method (2D MCM). The main difference between them is that the former uses fuzzy numbers as input where as the latter uses probability density functions as inputs. Kentel \& Aral (2005) proved that the 2D FMCA is more realistic than the pure 2D MCM in health risk assessment applications where probabilistic and possibility information may exist. A joint-propagation method (Baudrit et al 2007) is discussed for groundwater contamination risk assessment and compared to interval analysis and MC method. Integrated fuzzy-stochastic modelling (IFSM) (Heng et al 2008) approach is developed for assessing air pollution impacts towards asthma susceptibility. This approach also quantifies uncertainties associated with both source/medium conditions and evaluation criteria and thus assesses air pollution risk. Chen et al (2010) integrated fuzzy set theory with Monte Carlo method to study the uncertainties associated with produced water discharges and related regulated pollution criteria for the marine environment. An Integrated Simulation-Assessment Approach (ISAA) (Yang et al 2010) is developed to systematically tackle multiple uncertainties associated with hydrocarbon contaminant transport in subsurface and assessment of carcinogenic health risk by integrating Fuzzy Latin Hypercube Sampling (FLHS) and Fuzzy-rule-based Risk Assessment (FRRA) within a general risk assessment framework. Fuzzy Parametrized Probabilistic Analysis (FPPA) (Qin 2012) is a method for assessing risk associated with environmental pollution-control problems.

The above literature review admits that with the advancement of different types of uncertainty it has became a trend in coupling them into a single modelling platform . These types of modelling are often termed as Hybrid methods. The article proposes a hybrid method of uncertainty analysis. The SRSM is a purely probabilistic method of uncertainty analysis. Here an attempt is made to combine probability theory and fuzzy theory through the SRSM and to show its application in uncertainty analysis of atmospheric dispersion model as an hypothetical case study.

\section{The stochastic response surface method (SRSM)}

The SRSM is an extension of the classical deterministic response surface method (RSM), on which extensive discussion are made in Box \& Draper (1987) and Box et al (1978). The basic difference between the SRSM and the RSM is that in the former the inputs are random variables, whereas in the latter the inputs are deterministic variables. The SRSM is based on Polynomial Chaos Expansion (PCE) approach. The PCE approach has its foundation in the work by Wiener (1938), who represented a Gaussian process as an infinite series of the Hermite polynomial that takes a vector of random variables as arguments. The explanation of the SRSM is presented here through the PCE approach.

The PCE is the representation of a random variable, more generally a stochastic process, with an infinite series of orthogonal polynomials that take a vector of independent and identically distributed random variables, each with zero mean and unit variance, as arguments (Datta \& Kushwaha 2011). These random variables are referred as Standard Random Variables (srvs). The 
expansion uses a rescaled version of Hermite polynomials which correspond to a Gaussian or normal distribution. In SRSM, inputs are represented as functions of normal random variables, each having zero mean and unit variance. The same set of random variables that is used to represent input stochasticity can then be used for representation of outputs. The model output in terms of the srvs as multidimensional Hermite polynomial with unknown coefficients, as follows:

$$
\begin{array}{r}
y_{j}=a_{j, 0}+\sum_{i_{1}=1}^{n} a_{j, i_{1}} \Gamma_{1}\left(\zeta_{i_{1}}\right)+\sum_{i_{1}=1}^{n} \sum_{i_{2}=1}^{i_{1}} a_{j, i_{1} i_{2}} \Gamma_{2}\left(\zeta_{i_{1}}, \zeta_{i_{2}}\right) \\
+\sum_{i_{1}=1}^{n} \sum_{i_{2}=1}^{i_{1}} \sum_{i_{3}=1}^{i_{2}} a_{j, i_{1} i_{2} i_{3}} \Gamma_{3}\left(\zeta_{i_{1}}, \zeta_{i_{2}}, \zeta_{i_{3}}\right)+\ldots
\end{array}
$$

where $a_{j, i_{1}}, a_{j, i_{2}}, \cdots$ are unknown coefficients to be determined with respect to the specified model used for uncertainty analysis, and $\Gamma_{p}\left(\zeta_{i_{1}}, \cdots, \zeta_{i_{p}}\right)$ are defined to be multivariate Hermite polynomials in the $p$-dimensional sequence of uncorrelated standard normal random variables. The multivariate Hermite polynomial can be written as,

$$
\Gamma_{p}\left(\zeta_{i_{1}}, \cdots, \zeta_{i_{p}}\right)=(-1)^{p} e^{\frac{1}{2} \zeta^{T} \zeta} \frac{\partial^{p}}{\partial \zeta_{i_{1}} \cdots \partial \zeta_{i_{p}}} e^{-\frac{1}{2} \zeta^{T} \zeta}
$$

where $\zeta$ is the vector of $p$ identically independently distributed random normal variables $\left\{\zeta_{i_{k}}\right\}_{k=1}^{p}$.

The stochastic response surface described by Eq. (3) must be limited to a more reasonable size to allow for the evaluation of the model required. The limiting factor is the number of the uncertain parameter present in the system along with the chosen limit on the single variable representation of the polynomial basis. The space is limited to a finite number of terms $N$ given by

$$
N=\frac{(p+n) !}{p ! n !}
$$

where $p$ is the order of the polynomial and $n$ is the number of srvs used to represent the uncertainty in model inputs. Thus for simplicity, the Eq. (3) may be written as

$$
y_{i}=\sum_{i=0}^{N} \beta_{i} \Phi_{i}(\zeta)
$$

in which $\beta_{i}$ and $\Phi_{i}(\zeta)$ are identical to $a_{j, i_{1}}, a_{j, i_{2}}, \cdots$ and $\Gamma_{p}\left(\zeta_{i_{1}}, \cdots, \zeta_{i_{p}}\right)$, respectively.

The minimum numbers of srvs needed to represent the inputs is defined as the number of degrees of freedom in input uncertainty. In practice, in the theory of PCE, the minimum number of simulations required for generating the sample points of the uncertain inputs from the respective probability density functions depend on the order of the Hermite polynomial and the number of uncertain inputs. Since the model outputs are deterministic functions of model inputs, they have at most the same degree of freedom in uncertainty. So, the number of unknown coefficients to be determined for the model output can be explicitly written using Eq. (5) as

$$
N_{2}=1+2 n+\frac{n(n-1)}{2}, \text { for } 2^{\text {nd }} \text { order Hermite polynomial, }
$$




$$
N_{3}=1+3 n+\frac{3 n(n-1)}{2}+\frac{n(n-1)(n-2)}{6}, \text { for } 3^{\text {rd }} \text { order Hermite polynomial. }
$$

So, an explicit representation of $2^{\text {nd }}$ order polynomial chaos expansion for two and three uncertain inputs can be written using Eqs. (6-7) as

$$
\begin{gathered}
y_{2}=a_{0}+a_{1} \zeta_{1}+a_{2} \zeta_{2}+a_{3}\left(\zeta_{1}^{2}-1\right)+a_{4}\left(\zeta_{2}^{2}-1\right)+a_{5} \zeta_{1} \zeta_{2} \\
y_{3}=a_{0}+a_{1} \zeta_{1}+a_{2} \zeta_{2}+a_{3} \zeta_{3}+a_{4}\left(\zeta_{1}^{2}-1\right)+a_{5}\left(\zeta_{2}^{2}-1\right)+a_{6}\left(\zeta_{3}^{2}-1\right)+a_{7} \zeta_{1} \zeta_{2}+a_{8} \zeta_{1} \zeta_{3}+a_{9} \zeta_{2} \zeta_{3} .
\end{gathered}
$$

According to the number of uncertain model inputs, $n=2,3$ and 4 , the number of unknown coefficients to be determined in the polynomial chaos expansion can be obtained using Eqs. (7) and (8) as $\{6,10$ and 15$\}$ and $\{10,20$ and 35$\}$, respectively. Thus for two uncertain model inputs the second order polynomial needs six simulations to estimate the unknown coefficients. The estimation of coefficients of series expansions model inputs are sampled in terms of srvs and the model outputs at these sample points are used in Equation (3) to obtain a linear system of equation as $[\zeta]\{a\}=y$, from which coefficient vector $\{a\}$ can be solved using singular value decomposition.

For reference, the first few Hermite polynomials are given by

$$
H_{0}(\zeta)=1, H_{1}(\zeta)=\zeta, H_{2}(\zeta)=\zeta^{2}-1
$$

and the higher order Hermite polynomials can be generated using the recurrence relation given by

$$
H_{k+1}(\zeta)=\zeta H_{k}(\zeta)-k H_{k-1}(\zeta)
$$

\subsection{Representation of input distribution in terms of srvs}

Normal random variables are selected as srvs as they have been extensively studied and their functions are typically well-behaved. Here, the srvs are selected from a set of independent, identically distributed normal random variables. Each $\zeta_{i}$ has zero mean and unit variance. With the advent of newer uncertainty theories such as fuzzy set theory, evidence theory, possibility theory, random set theory and others, it is necessary to revisit the different kinds of uncertainty inherent in different kinds of modelling. When some uncertain parameters are represented as probability distributions while others are represented as possibility distributions, a transformation method will then be used to propagate these hybrid uncertainties through different models. The SRSM is accomplished to incorporate fuzzy uncertainties as model inputs. This is performed by discretizing the fuzzy parameter into $\alpha$-cuts, say $\alpha=[0,1 / m, 2 / m, \cdots, 1], m$ is any natural number and each of these $\alpha$-cuts represent an interval. Without loss of generality the underlying uncertainty can be very well expressed by a uniform distribution. Suppose that only thing known about a variable $X$ is that it can take values between 0 and 1 . Apparently, a uniform distribution $U(0,1)$ is an obvious choice (Brandimarte 2011; Nguyen 2012). The $\alpha$-cut of a fuzzy number gives the possible information of a variable with some degree of membership and its distribution is often unknown. In such a situation uniform distribution around the bounds of the $\alpha$-cut is obvious choice. Thus each $\alpha$-cut of the fuzzy number is finally transformed into srvs. Rather than 
discussing about the common univariate distribution following a univariate continuous probability density function, the following are the relationship between transformed and univariate probability density function for different distribution.

Uniform distribution $(a, b): X=a+(b-a)\left(\frac{1}{2}+\frac{1}{2} \operatorname{erf}(\zeta / \sqrt{2})\right)$.

Normal distribution $(\mu, \sigma): X=\mu+\sigma \zeta$.

Lognormal distribution $(\mu, \sigma): X=\exp (\mu+\sigma \zeta)$.

Gamma distribution $(a, b): X=a b\left(\zeta \sqrt{\frac{1}{9 a}}+1-\frac{1}{9 a}\right)$.

Exponential distribution $\lambda: X=-\frac{1}{\lambda}\left(\frac{1}{2}+\frac{1}{2} \operatorname{erf}(\zeta / \sqrt{2})\right)$.

Triangular fuzzy number $(a, b, c): X_{\alpha}=p+(q-p)\left(\frac{1}{2}+\frac{1}{2} \operatorname{erf}(\zeta / \sqrt{2})\right)$,

where, $p=a+\alpha(b-a), q=c+\alpha(b-c)$ and $\alpha=\left[0, \frac{1}{p}, \frac{2}{p}, \cdots, 1\right], p$ being a natural number.

Trapezoidal fuzzy number $(a, b, c, d): X_{\alpha}=p+(q-p)\left(\frac{1}{2}+\frac{1}{2} \operatorname{erf}(\zeta / \sqrt{2})\right)$,

where, $p=a+\alpha(b-a), q=d+\alpha(c-d)$ and $\alpha=\left[0, \frac{1}{p}, \frac{2}{p}, \cdots, 1\right], p$ being a natural number.

\subsection{An illustration of the application of the SRSM under aleatory and epistemic uncertainties}

As an illustration, consider a computational model with two independent uncertain inputs $X_{1}$ and $X_{2}$, and output $Y_{\alpha, 2}$, where the inputs are given by

$$
X_{1}=\operatorname{Normal}(\mu, \sigma), X_{2}=\text { Triangular fuzzy number }(a, b, c) .
$$

The input uncertain variables can be represented by srvs $\zeta_{1}$ and $\zeta_{2}$ as follows:

$$
\begin{aligned}
& X_{1}=\mu+\sigma \zeta_{1}, X_{\alpha, 2}=p+(q-p)\left(\frac{1}{2}+\frac{1}{2} \operatorname{erf}\left(\zeta_{2} / \sqrt{2}\right)\right), \text { where } p=a+\alpha(b-a), \\
& q=c+\alpha(b-c) \text { and } \alpha=\left[0, \frac{1}{m}, \frac{2}{m}, \cdots, 1\right], m \text { being a natural number, }
\end{aligned}
$$

where $X_{\alpha, 2}$ is transformed input variable in terms of srvs, $\zeta_{2}$ at a particular $\alpha$ level. A second order polynomial approximation for $Y_{\alpha, 2}$ in terms of $\zeta_{1}$ and $\zeta_{2}$ is given by

$$
y_{\alpha, 2}=a_{\alpha, 0}+a_{1} \zeta_{1}+a_{\alpha, 2} \zeta_{2}+a_{\alpha, 3}\left(\zeta_{1}^{2}-1\right)+a_{\alpha, 4}\left(\zeta_{2}^{2}-1\right)+a_{\alpha, 5} \zeta_{1} \zeta_{2} .
$$

In order to estimate the 6 unknown coefficients, for response output, the inputs are sampled as $\left(x_{1,1}, x_{1,2}, \cdots, x_{1,6}\right)$ and $\left(x_{\alpha, 2,1}, x_{\alpha, 2,2}, \cdots, x_{\alpha, 2,6}\right)$. Then the outputs at these sample points are 
obtained as $y_{\alpha, 1,1}, y_{\alpha, 1,2}, \cdots, y_{\alpha, 1,6}$ and used to calculate the coefficients $a_{\alpha, 0}, a_{\alpha, 1}, \cdots, a_{\alpha, 5}$ by solving the following linear equations through singular value decomposition. Let

$$
M=\left[\begin{array}{llllll}
1 & \zeta_{1,1} & \zeta_{2,1} & \zeta_{1,1}^{2}-1 & \zeta_{2,1}^{2}-1 & \zeta_{1,1} \zeta_{2,1} \\
1 & \zeta_{1,2} & \zeta_{2,2} & \zeta_{1,2}^{2}-1 & \zeta_{2,2}^{2}-1 & \zeta_{1,2} \zeta_{2,2} \\
1 & \zeta_{1,3} & \zeta_{2,3} & \zeta_{1,3}^{2}-1 & \zeta_{2,3}^{2}-1 & \zeta_{1,3} \zeta_{2,3} \\
1 & \zeta_{1,4} & \zeta_{2,4} & \zeta_{1,4}^{2}-1 & \zeta_{2,4}^{2}-1 & \zeta_{1,4} \zeta_{2,4} \\
1 & \zeta_{1,5} & \zeta_{2,5} & \zeta_{1,5}^{2}-1 & \zeta_{2,5}^{2}-1 & \zeta_{1,5} \zeta_{2,5} \\
1 & \zeta_{1,6} & \zeta_{2,6} & \zeta_{1,6}^{2}-1 & \zeta_{2,6}^{2}-1 & \zeta_{1,6} \zeta_{2,6}
\end{array}\right]
$$

which can be calculated from the values of $\zeta$ 's at each sample point whereas $y_{\alpha, 1, i}$ are the corresponding model outputs obtained from the linear system

$$
M \times\left[a_{\alpha, 0}, a_{\alpha, 1}, \cdots, a_{\alpha, 5}\right]^{T}=\left[y_{\alpha, 1,1}, y_{\alpha, 1,2}, \cdots, y_{\alpha, 1,6}\right]^{T} .
$$

Now, the coefficients are estimated, the distribution of $Y_{\alpha, 2}$ is fully described by polynomial chaos expansion as shown in Eq. (13). The whole process is repeated for all the $\alpha$-cut taken. Statistical properties from response can be obtained by performing a large number of MonteCarlo simulation at each level of $\alpha$ 's.

\section{A case study}

\subsection{Problem statement}

A case study is made by considering the example cited in Abrahamsson (2002), the problem involves a simplified pressurized ammonia storage facility, consisting of a pressurized tank and 3 meters of pipeline. The assessment will consider the release of pressurized ammonia to the surroundings, and the assessment end-points are to determine concentration of ammonia at a geographical location. A dispersion model is used for estimating the concentration of ammonia at a specific geographic point. The average concentration of ammonia at a particular location, following a discharge is given by the following expression.

$$
C=\frac{Q}{\pi \sigma_{y} \sigma_{z} U},
$$

where,

$C \quad$ Concentration of ammonia $\left(\mathrm{kg} / \mathrm{m}^{3}\right)$.

$Q \quad$ Mass discharge/release rate $(\mathrm{kg} / \mathrm{sec})$.

$\sigma_{y}, \sigma_{z}$ Dispersion coefficients (m).

$U \quad$ Wind speed $(\mathrm{m} / \mathrm{sec})$.

The equation of mass discharge rate is given by

$$
Q=C_{d} A \sqrt{\frac{2\left(P_{o}-P_{a}\right)}{V_{f}}},
$$


where,

$C_{d}$ Discharge coefficient.

A Area of the hole $\left(\mathrm{m}^{2}\right)$.

$P_{o}$ Pressure in the tank $\left(\mathrm{N} / \mathrm{m}^{2}\right)=5 \times 10^{5}$.

$P_{a}$ Atmospheric pressure $\left(\mathrm{N} / \mathrm{m}^{2}\right)=1 \times 10^{5}$.

$V_{f}$ Specific volume of liquid $\left(\mathrm{m}^{3} / \mathrm{kg}\right)=1 / 617$.

The state of art models related to atmospheric dispersion is deterministic in nature. The Eq. (14) describing the average concentration of ammonia, as a case study to support our approach of combining aleatory and epistemic uncertainty, presents the simplified version of Gaussian plume model under the conditions of plume centerline (coordinate, $y=0$ ), ground level (vertical coordinate, $z=0$ ) and ground release. Average concentration as depicted in Eq. (14) is a function of the release quantity, the horizontal dispersion coefficient, the vertical dispersion coefficient and the wind speed. In a deterministic model, all these input parameters of the model are crisp, but in real practice, one can never have deterministic value of all these parameters. For example, wind speed is obviously a probabilistic parameter due to its random variation over a certain cycle. On the other hand, the horizontal dispersion and the vertical dispersion coefficients are dependent on the stability class of the wind, which further depends on solar radiation, wind speed, etc. The measured value of the solar radiation and the wind speed averaged over a certain cycle by any instrument is obtained in the form of a range value, and these range value can be possibly tackled by using the theory of fuzzy set. Therefore, if a realistic situation is considered the output cannot be a crisp value, and it is also a fact that, one cannot take any decision if only crisp value of the output as well as the input are optioned. Decision making in the field of atmospheric dispersion modelling has to be taken in presence of uncertainty which is a combination of both the aleatory (probabilistic) and epistemic (lack of knowledge or incomplete information).

In view of this reality, the present model in which the approach of handling both the uncertainties have been presented, will facilitate the decision makers in the said field to take a decision on the quality of the air if pollutants get released in the event of the failure of a system or mal operation during normal practice. For reader's information, it is worth to mention that the atmospheric dispersion models in practice always dictate about the health status of the atmosphere with the help of computed air concentration, without quantifying its uncertainty. But uncertainty of the air concentration is always desired to have a safety measure of any nuclear or industrial facility.

\subsection{Input parameters and the methodology}

Randomness behaviour of uncertain input variable is described by a probability density function and this is basically constructed by adopting a statistical fitting technique. Very often, in practice, parameters of the probability distribution are estimated using available or given experimental data by maximum likelihood method. Implementation of this procedure results the specific probability density function of the input random uncertain variable. On the other hand, imprecise uncertain variables are expressed in terms of a triangular fuzzy numbers, 'in the sense that linguistic phrase of this kind of' uncertainty is very often practised by saying that 'the value of the area of hole is around $A \mathrm{~m}^{2}$ '. Now this 'around $A$ ' is further expressed by giving a lower and a upper bounds, and accordingly then one have, 〈lower bound of $A$, crisp value of $A$, upper bound of $A$ 〉 and this comprises a triangular fuzzy number which express the epistemic uncertainty of the uncertain variable $A$. 
Wind speed, discharge coefficient and area of hole are considered to be uncertain variables. Table 1 depicts the uncertainty of the input parameters at different stability categories B, D, E and $\mathrm{F}$. The wind speed $U$ under different stability category constitute of probability distributions similarly the discharge coefficient $C_{d}$ is taken to be uniformly distributed and the parameter area of hole $A$ is considered uncertain in the sense of fuzzy theory and is considered as triangular fuzzy number. The average ammonia concentration is evaluated at downwind distance $x=300 \mathrm{~m}$ and the crosswind distance $y=0 \mathrm{~m}$. The dispersion coefficient in the horizontal and the crosswind direction are calculated from the Eimutis \& Konicek table in Eimutis \& Konicek (1972). The fuzzy parameter area of hole $A$ is discretized into 6 number of $\alpha$-cuts that is $p=5$ which is chosen arbitrarily. Then each of these $\alpha$-cuts are expressed as srvs, that is, uniformly distributed srvs. Total number of simulations chosen for sampling the random parameters here based on the order of the polynomial to be fitted and the number of uncertain inputs. The number of uncertain parameters are 3 and the considered order of Hermite polynomial is 2, so the numbers of unknown coefficients to be determined in the polynomial chaos expansion is 10 . Number of repeations of the whole computation depends on the numbers of $\alpha$-cuts chosen for the fuzzy inputs. Since, the number of $\alpha$-cuts chosen is 6 , the computation repeats for 6 times. At each repeation, that is, for a particular $\alpha$-cut level, of the computation 10 numbers of coefficients of the response are solved using singular value decomposition. Finally, for all the $\alpha$-cut levels $6 \times 10$ coefficients are solved by using single value decomposition. Statistics of the response surface at the various $\alpha$-cut levels are thus constructed using a large number of Monte-Carlo simulations.

\subsection{Results and discussions}

The conventional SRSM is a probabilistic process where the inputs are always aleatory. However, the present methodology tries to address the uncertainty due aleatory and epistemic uncertainties. This methodology is applicable, whether the inputs are aleatory or epistemic, however the conventional SRSM is only applicable when the information about the inputs are aleatory. As mentioned earlier, all the information about input parameters may not be always probabilistic or may have uncertainties due to lack of measurement point and over calibration or inaccurate expert judgement. In such case the conventional SRSM is not applicable. But the present methodology can be very well applied to such situation where fuzzy numbers exist as epistemic uncertainties.

Uncertainty analysis is carried out using the SRSM and the stochastic response surface of ammonia concentration is simulated at different $\alpha$-cuts. The values of the coefficients of the response surface for various $\alpha$-cuts and for stability categories B, C, D and E are given in tables 2, 3, 4 and 5, respectively. A second order polynomial chaos expansion of ammonia concentration is generated at each $\alpha$-cut. For the stability category $\mathrm{F}$ in the table 5 the first column

Table 1. Information for uncertain parameters.

\begin{tabular}{lc}
\hline Parameters & Values \\
\hline Wind speed $U,($ Stability B) & Normal $[4,0.4](\mathrm{m} / \mathrm{sec})$ \\
Wind speed $U,($ Stability D) & Normal $[5,1.5](\mathrm{m} / \mathrm{sec})$ \\
Wind speed $U,($ Stability E) & Normal $[4,0.4](\mathrm{m} / \mathrm{sec})$ \\
Wind speed $U,($ Stability F) & Uniform $[1,2](\mathrm{m} / \mathrm{sec})$ \\
Discharge coefficient $C_{d}$ & Uniform $[0.7,0.9]$ \\
Area of hole $A$ & $\mathrm{tfn}\langle 0.0012,0.00185,0.0025\rangle\left(\mathrm{m}^{2}\right)$. \\
\hline
\end{tabular}

tfn - Triangular fuzzy number 
Table 2. Coefficients of response polynomial for the various $\alpha$-cuts level under the stability category B.

\begin{tabular}{lcccccc}
\hline & $\alpha=0.0$ & $\alpha=0.2$ & $\alpha=0.4$ & $\alpha=0.6$ & $\alpha=0.8$ & $\alpha=1.0$ \\
\hline$a_{0}$ & 01849.0376 & 01844.3953 & 01839.7529 & 01835.1106 & 01830.4683 & 01825.8260 \\
$a_{1}$ & 00380.7306 & 00305.1876 & 00229.6447 & 00154.1017 & 00078.5588 & 00003.0159 \\
$a_{2}$ & -0121.3199 & -0131.4698 & -0141.61972 & -0151.7696 & -0161.9194 & -0172.0693 \\
$a_{3}$ & 00018.3564 & 00042.0024 & 00065.6484 & 00089.2944 & 00112.9404 & 00136.5864 \\
$a_{4}$ & 00058.5818 & 00049.0799 & 00039.5779 & 00030.0759 & 00020.5740 & 00011.0720 \\
$a_{5}$ & 00119.4595 & 00101.3484 & 00083.2372 & 00065.1260 & 00047.0149 & 00028.9037 \\
$a_{6}$ & -0062.5325 & -0049.9506 & -0037.3687 & -0024.7868 & -0012.2049 & 00000.3770 \\
$a_{7}$ & -0134.9909 & -0109.4779 & -0083.9649 & -0058.4519 & -0032.9389 & -0007.4260 \\
$a_{8}$ & -0091.9802 & -0081.4068 & -0070.8334 & -0060.2601 & -0049.6867 & -0039.1133 \\
$a_{9}$ & -0025.2772 & -0019.7766 & -0014.2761 & -0008.7754 & -0003.2749 & 00002.2256 \\
\hline
\end{tabular}

Table 3. Coefficients of response polynomial for the various $\alpha$-cuts level under the stability category D.

\begin{tabular}{ccccccc}
\hline & $\alpha=0.0$ & $\alpha=0.2$ & $\alpha=0.4$ & $\alpha=0.6$ & $\alpha=0.8$ & $\alpha=1.0$ \\
\hline$a_{0}$ & 07207.3914 & 07161.9173 & 07116.4431 & 07070.9690 & 07025.4948 & 06980.0207 \\
$a_{1}$ & 01653.9969 & 01353.6105 & 01053.2240 & 00752.8376 & 00452.4512 & 00152.0648 \\
$a_{2}$ & -1629.0261 & -1744.7611 & -01860.4961 & -01976.2312 & -02091.9663 & -2207.7013 \\
$a_{3}$ & 00630.9017 & 00572.8878 & 00514.8739 & 00456.8599 & 00398.8460 & 00340.8321 \\
$a_{4}$ & 00351.3703 & 00243.8312 & 00136.2922 & 00028.7532 & -0078.7858 & -0186.3249 \\
$a_{5}$ & 00249.1773 & 00265.6040 & 00282.0306 & 00298.4572 & 00314.8838 & 00331.3105 \\
$a_{6}$ & -0060.7011 & -0069.7562 & -0078.81130 & -0087.8664 & -0096.9215 & -0105.9766 \\
$a_{7}$ & 00387.8209 & 00262.1281 & 00136.4353 & 00010.7425 & -0114.9502 & -0240.6430 \\
$a_{8}$ & 00443.4241 & 00373.8080 & 00304.1920 & 00234.5760 & 00164.9599 & 00095.3439 \\
$a_{9}$ & 00681.3018 & 00520.7143 & 00360.1268 & 00199.5393 & 00038.9518 & -0121.6356 \\
\hline
\end{tabular}

Table 4. Coefficients of response polynomial for the various $\alpha$-cuts level under the stability category E.

\begin{tabular}{ccccccc}
\hline & $\alpha=0.0$ & $\alpha=0.2$ & $\alpha=0.4$ & $\alpha=0.6$ & $\alpha=0.8$ & $\alpha=1.0$ \\
\hline$a_{0}$ & 16359.4832 & 16423.5991 & 16487.7149 & 16551.8307 & 16615.9466 & 16680.0624 \\
$a_{1}$ & 03879.1608 & 03190.5227 & 02501.8847 & 01813.2466 & 01124.6086 & 00435.9705 \\
$a_{2}$ & -1432.5703 & -01384.1971 & -1335.8240 & -1287.4509 & -1239.0778 & -1190.7047 \\
$a_{3}$ & 00967.9851 & 01104.9898 & 01241.9945 & 01378.9992 & 01516.0039 & 01653.0086 \\
$a_{4}$ & 00590.4612 & 00487.5045 & 00384.5477 & 00281.5909 & 00178.6341 & 00075.6773 \\
$a_{5}$ & 00123.4928 & 00089.9591 & 00056.4254 & 00022.8917 & -0010.6419 & -0044.1756 \\
$a_{6}$ & -0318.5054 & -0148.2563 & 00021.9927 & 00192.2419 & 00362.4910 & 00532.7401 \\
$a_{7}$ & 00364.6954 & 00460.3804 & 00556.0653 & 00651.7503 & 00747.4352 & 00843.1202 \\
$a_{8}$ & 00329.1031 & 00393.3888 & 00457.6746 & 00521.9604 & 00586.2461 & 00650.5319 \\
$a_{9}$ & -0313.3557 & -0225.5448 & -0137.7341 & -0049.9232 & 00037.8875 & 00125.6983 \\
\hline
\end{tabular}


Table 5. Coefficients of response polynomial for the various $\alpha$-cuts level under the stability category F.

\begin{tabular}{lcccccc}
\hline & $\alpha=0.0$ & $\alpha=0.2$ & $\alpha=0.4$ & $\alpha=0.6$ & $\alpha=0.8$ & $\alpha=1.0$ \\
\hline$a_{0}$ & 107518.359 & 107027.361 & 106536.363 & 106045.365 & 105554.366 & 105063.368 \\
$a_{1}$ & 20202.4339 & 16353.9881 & 12505.5422 & 08657.0963 & 04808.6504 & 00960.2046 \\
$a_{2}$ & -19178.428 & -19396.338 & -19614.247 & -19832.157 & -20050.066 & -20267.976 \\
$a_{3}$ & 06115.3554 & 06604.8067 & 07094.2580 & 07583.7093 & 08073.1607 & 08562.6120 \\
$a_{4}$ & -4396.1782 & -3630.9806 & -2865.7830 & -2100.5853 & -1335.3877 & -0570.1901 \\
$a_{5}$ & 05754.0893 & 05441.0179 & 05127.9464 & 04814.8750 & 04501.8035 & 04188.7321 \\
$a_{6}$ & 04139.9984 & 03764.6865 & 03389.3747 & 03014.0629 & 02638.7511 & 02263.4392 \\
$a_{7}$ & -8007.0406 & -6779.9990 & -5552.9574 & -4325.9158 & -3098.8742 & -1871.8326 \\
$a_{8}$ & -0154.8431 & -0802.9014 & -1450.9596 & -2099.0179 & -2747.0761 & -3395.1344 \\
$a_{9}$ & 05616.7170 & 04105.1052 & 02593.4933 & 01081.8815 & -0429.7302 & -1941.3420 \\
\hline
\end{tabular}

and the last column gives the response polynomial of ammonia concentration at the $\alpha$-cut level 0 and 1 , respectively as follows:

$$
\begin{aligned}
& y_{0,3}=107518.359+20202.4339 \zeta_{1}-19178.428 \zeta_{2} \\
&+6115.3554 \zeta_{3}-4396.1782\left(\zeta_{1}^{2}-1\right)+5754.0893\left(\zeta_{2}^{2}-1\right) \\
&+4139.9984\left(\zeta_{3}^{2}-1\right)-8007.0406 \zeta_{1} \zeta_{2}-154.8431 \zeta_{1} \zeta_{3}+5616.717 \zeta_{2} \zeta_{3} \\
& y_{1,3}=105063.368+960.2046 \zeta_{1}-20267.976 \zeta_{2} \\
&+ 8562.612 \zeta_{3}-570.1901\left(\zeta_{1}^{2}-1\right)+4188.7321\left(\zeta_{2}^{2}-1\right) \\
&+2263.4392\left(\zeta_{3}^{2}-1\right)-1871.8326 \zeta_{1} \zeta_{2}-3395.1344 \zeta_{1} \zeta_{3}-1941.342 \zeta_{2} \zeta_{3} .
\end{aligned}
$$

Eventually, response surface can be obtained at other $\alpha$-cuts and for other stability categories.

Now the response surface are generated for all the stability categories and at different level of $\alpha$-cuts, statistical properties of ammonia concentration are evaluated from these response surfaces. A large number of simulations, say 1500, is used to generate the cumulative distribution functions. These distribution functions of ammonia concentration at all the $6 \alpha$-cuts for the stability categories B, D, E and F are shown in figures 1, 2, 3 and 4, respectively. Percentiles are best representation of uncertainty; hence $5^{\text {th }}$ and $95^{\text {th }}$ percentiles of the cumulative distribution function of ammonia concentration are obtained and depicted in tables 6, 7, 8 and 9. The basic aim of the problem is to generate a response surface in terms of cumulative distribution functions of the ammonia concentration $\left(\mathrm{mg} / \mathrm{m}^{3}\right)$ at different $\alpha$-cuts. So, that different statistical properties can be obtained from the cumulative distribution functions at various $\alpha$-cuts. Accordingly, uncertainty analyses of ammonia concentration at downwind distance $300 \mathrm{~m}$ are obtained. In order to quantify these uncertainties, the $5^{\text {th }}$ and the $95^{\text {th }}$ percentiles are obtained from the cumulative distribution functions at various $\alpha$-cuts for the different stability categories.

As per regulatory practice under safe design it is always essential to consider a conservative estimate and conservative estimate generally addresses a maximum value, therefore it is mandatory to know that for which weather category maximum uncertainty of air pollutant concentration results. The $5^{\text {th }}$ and the $95^{\text {th }}$ percentiles in tables $6,7,8$ and 9 , indicate that uncertainty 


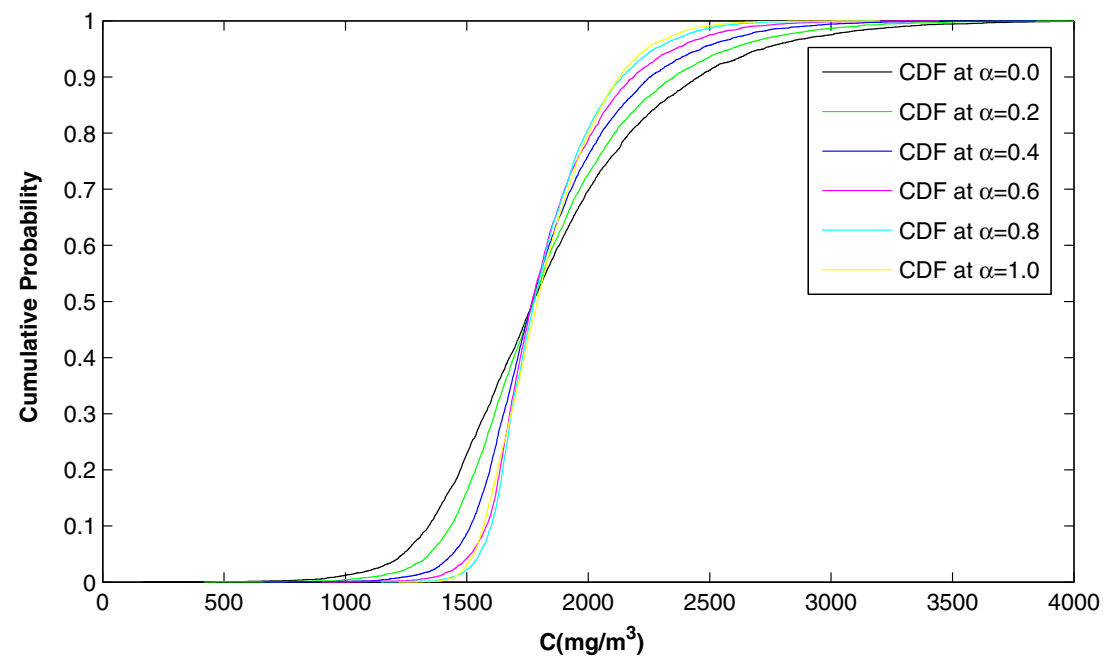

Figure 1. Cumulative distribution functions of ammonia concentration $\left(\mathrm{mg} / \mathrm{m}^{3}\right)$ at different level of $\alpha$ cuts by SRSM for stability B.

increases through B (moderately unstable) to F (very stable) for all the chosen $\alpha$-cuts. Accordingly, the present methodology of uncertainty modelling of atmospheric dispersion indicates that safe design of the specific facility (either nuclear or chemical) should be with respect to extremely stable category of weather. Hence, utmost care should be taken for discharge of toxic or radioactive effluent under the stability category $\mathrm{F}$.

Uncertainty in the domain of probability theory is expressed in terms of confidence interval and is always expressed as $\langle x\rangle \pm k \sigma$, where, $\langle x\rangle$ presents the average (crisp) value, $k$ signifies the critical value (area under the curve representing the probability distribution, in case of a normal distribution, for $95 \%$ confidence interval, $k=1.96$ ) and $\sigma$ represents the standard deviation.

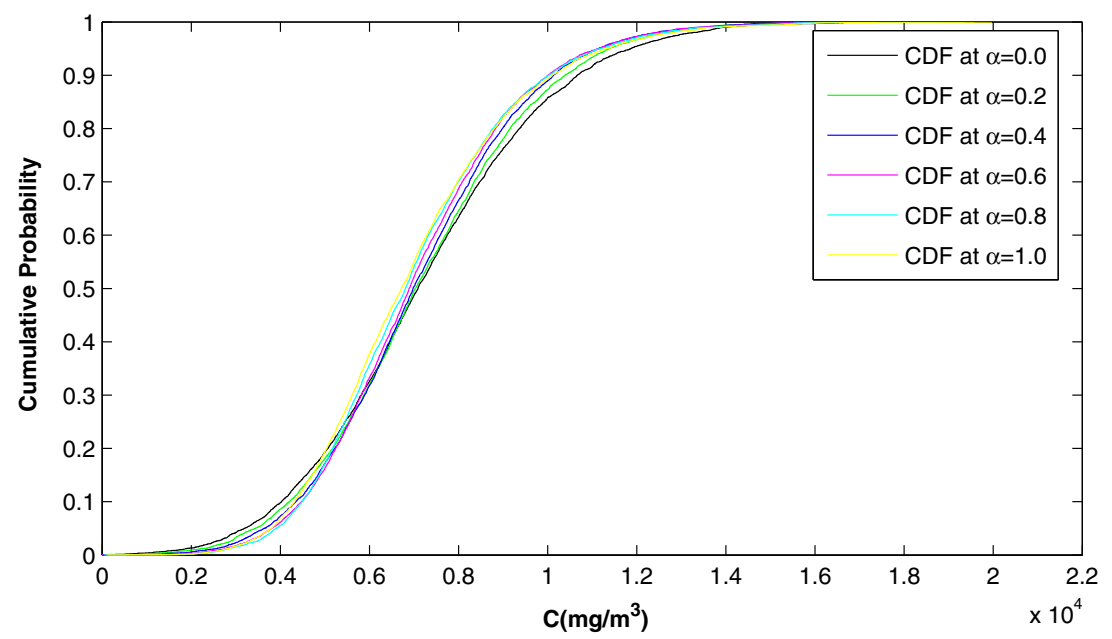

Figure 2. Cumulative distribution functions of ammonia concentration $\left(\mathrm{mg} / \mathrm{m}^{3}\right)$ at different level of $\alpha$ cuts by SRSM for stability D. 


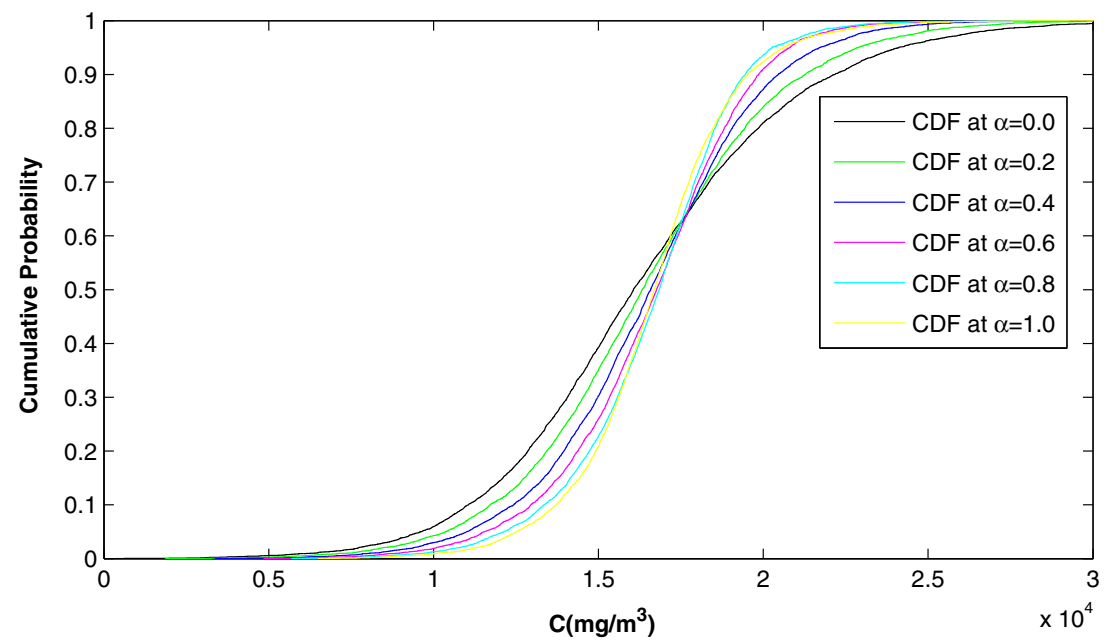

Figure 3. Cumulative distribution functions of ammonia concentration $\left(\mathrm{mg} / \mathrm{m}^{3}\right)$ at different level of $\alpha$ cuts by SRSM for stability E.

But the uncertainty in case of lack of information of an input parameter of a model is handled by using fuzzy set, since fuzzy set describes the vagueness or lack of information. Now, following the definition of a fuzzy set, practice is to construct $\alpha$-cut of the system and since $\alpha$-cut represents an interval in the real axis, uncertainty of the system is presented in terms of an interval that is nothing but a specific $\alpha$-cut value. Maximum uncertainty of the system is obviously corresponds to zero $\alpha$-cut value. According to practice in the field of fuzzy mathematics, uncertainty is expressed for zero $\alpha$-cut value of the system, but one can also take any $\alpha$-cut value to express

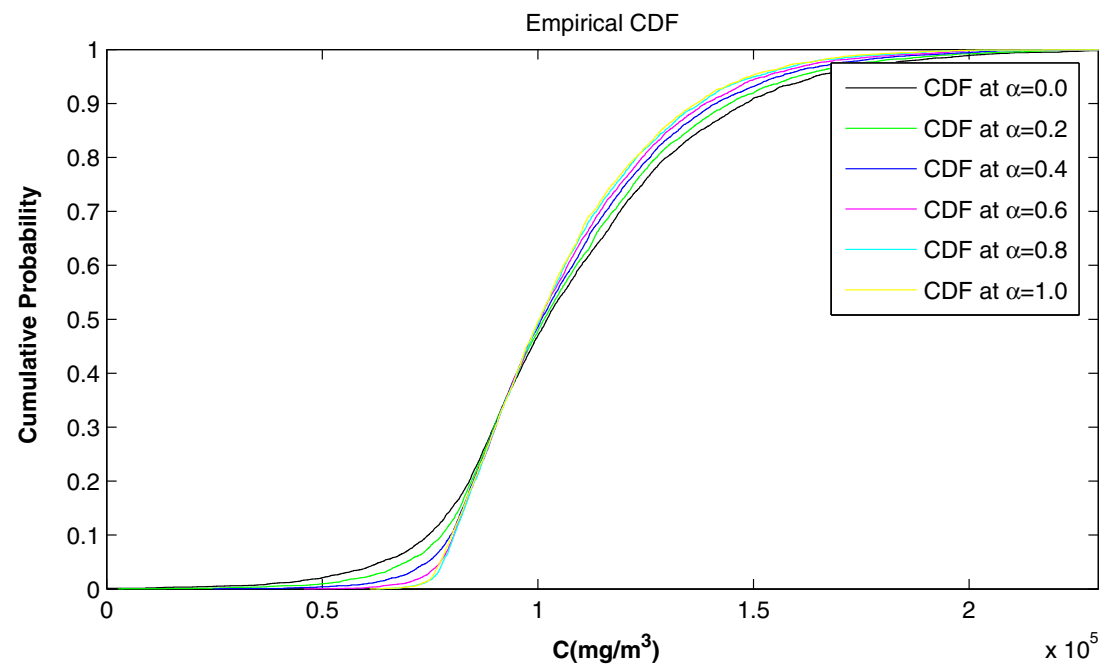

Figure 4. Cumulative distribution functions of ammonia concentration $\left(\mathrm{mg} / \mathrm{m}^{3}\right)$ at different level of $\alpha$ cuts by SRSM for stability F. 
Table 6. $5^{\text {th }}$ and $95^{\text {th }}$ percentiles of the ammonia concentration $\left(\mathrm{mg} / \mathrm{m}^{3}\right)$ at downwind distance $300 \mathrm{~m}$ under the stability category B by SRSM at different $\alpha$-cut levels from 0.0 to 1.0.

\begin{tabular}{lcc}
\hline & $5^{\text {th }}$ & $9^{\text {th }}$ \\
\hline $\operatorname{SRSM} \alpha=0.0$ & 1237.0497 & 2721.6446 \\
$\operatorname{SRSM} \alpha=0.2$ & 1344.5268 & 2590.4037 \\
$\operatorname{SRSM} \alpha=0.4$ & 1441.6850 & 2464.5767 \\
$\operatorname{SRSM} \alpha=0.6$ & 1516.1281 & 2359.3792 \\
$\operatorname{SRSM} \alpha=0.8$ & 1551.8207 & 2272.0581 \\
$\operatorname{SRSM} \alpha=1.0$ & 1528.1062 & 2240.7093 \\
\hline
\end{tabular}

Table 7. $5^{\text {th }}$ and $95^{\text {th }}$ percentiles of the ammonia concentration $\left(\mathrm{mg} / \mathrm{m}^{3}\right)$ at downwind distance $300 \mathrm{~m}$ under the stability category D by SRSM at different $\alpha$-cut levels from 0.0 to 1.0 .

\begin{tabular}{lcc}
\hline & $5^{\text {th }}$ & $9^{\text {th }}$ \\
\hline $\operatorname{SRSM} \alpha=0.0$ & 3195.9334 & 11872.0682 \\
$\operatorname{SRSM} \alpha=0.2$ & 3438.0222 & 11389.3320 \\
$\operatorname{SRSM} \alpha=0.4$ & 3643.6253 & 11132.1479 \\
$\operatorname{SRSM} \alpha=0.6$ & 3814.7037 & 11171.6992 \\
$\operatorname{SRSM} \alpha=0.8$ & 3908.6255 & 11194.8591 \\
$\operatorname{SRSM} \alpha=1.0$ & 3799.8296 & 11312.6081 \\
\hline
\end{tabular}

Table 8. $5^{\text {th }}$ and $95^{\text {th }}$ percentiles of the ammonia concentration $\left(\mathrm{mg} / \mathrm{m}^{3}\right)$ at downwind distance $300 \mathrm{~m}$ under the stability category E by SRSM at different $\alpha$-cut levels from 0.0 to 1.0.

\begin{tabular}{lcc}
\hline & $5^{\text {th }}$ & $9^{\text {th }}$ \\
\hline $\operatorname{SRSM} \alpha=0.0$ & 9613.7179 & 24111.5851 \\
$\operatorname{SRSM} \alpha=0.2$ & 10374.7193 & 22872.7412 \\
$\operatorname{SRSM} \alpha=0.4$ & 11002.5842 & 21715.0012 \\
$\operatorname{SRSM} \alpha=0.6$ & 11527.2709 & 20805.7029 \\
$\operatorname{SRSM} \alpha=0.8$ & 12137.4413 & 20267.9323 \\
$\operatorname{SRSM} \alpha=1.0$ & 12530.3296 & 20638.9835 \\
\hline
\end{tabular}

Table 9. $5^{\text {th }}$ and $95^{\text {th }}$ percentiles of the ammonia concentration $\left(\mathrm{mg} / \mathrm{m}^{3}\right)$ at downwind distance $300 \mathrm{~m}$ under the stability category F by SRSM at different $\alpha$-cut levels from 0.0 to 1.0.

\begin{tabular}{lcc}
\hline & $5^{\text {th }}$ & $9^{\text {th }}$ \\
\hline $\operatorname{SRSM} \alpha=0.0$ & 63720.4562 & 164217.2205 \\
$\operatorname{SRSM} \alpha=0.2$ & 69559.2123 & 160201.9260 \\
$\operatorname{SRSM} \alpha=0.4$ & 74467.4913 & 156083.3177 \\
$\operatorname{SRSM} \alpha=0.6$ & 77420.9970 & 152954.7499 \\
$\operatorname{SRSM} \alpha=0.8$ & 77990.1796 & 150527.4844 \\
$\operatorname{SRSM} \alpha=1.0$ & 77386.7157 & 149179.9540 \\
\hline
\end{tabular}


it, which can be agreed as an arbitrary. That is why one always tries to look for a method of quantification of uncertainty in which this arbitrariness can be avoided.

As the model is implemented under the presence of fuzzy uncertainty and random uncertainty under the SRSM, it is seen that cumulative distribution functions are generated at each level of $\alpha$ cuts. Statistical inference such as parametric estimation can be made about the parameters at each level of $\alpha$. The estimation to be made depends on consideration of the $\alpha$-cuts which ultimately depends on the decision-maker. A pessimistic decision-maker would choose the estimations at $\alpha=0$, whereas a optimistic decision-maker would choose the estimation at $\alpha=1$. For the stable category $\mathrm{F}$ a pessimistic decision-maker concludes that $5^{\text {th }}$ and $95^{\text {th }}$ percentiles of pollutant concentration are at $63720.4562 \mathrm{mg} / \mathrm{m}^{3}$ and $164217.2205 \mathrm{mg} / \mathrm{m}^{3}$, respectively. Whereas a optimistic decision-maker concludes that $5^{\text {th }}$ and $95^{\text {th }}$ percentiles of pollutant concentration are at $77386.7157 \mathrm{mg} / \mathrm{m}^{3}$ and $149179.9540 \mathrm{mg} / \mathrm{m}^{3}$, respectively. Also a pessimistic decision-maker would interprete that $95 \%$ of the ammonia concentration scored $164217.2205 \mathrm{mg} / \mathrm{m}^{3}$ or below and $5 \%$ of the ammonia concentration scored $63720.4562 \mathrm{mg} / \mathrm{m}^{3}$ or below. Further, a optimistic decision-maker would interprete that $95 \%$ of the ammonia concentration scored 149179.9540 $\mathrm{mg} / \mathrm{m}^{3}$ or below and $5 \%$ of the ammonia concentration scored $77386.7157 \mathrm{mg} / \mathrm{m}^{3}$ or below.

\section{Conclusion}

The conventional SRSM is a probabilistic method where the inputs are aleatory uncertainties. However, in the present methodology the SRSM is modified to incorporate aleatory and epistemic uncertainties. The aleatory uncertainties are probability density functions and epistemic uncertainties are fuzzy numbers. The inputs to the SRSM are always srvs, hence the fuzzy numbers are discretized into $\alpha$-cuts and each $\alpha$-cut is transformed into srvs. Hence a methodology of uncertainty analysis of a system under aleatory and epistemic uncertainties has been developed. The methodology is being demonstrated with the help of atmospheric dispersion model at the ground level for the atmospheric conditions B, D, E and F. It has been evident that ammonia concentration at the stability category $\mathrm{F}$ is higher than the other categories. Hence utmost care should be taken in modelling a situation under this category. The model output are demonstrated with the help of polynomial chaos expansions at different level of $\alpha$-cuts. Also, it has been evident that one can take different decision at different level of $\alpha$-cut. Any statistical properties could be generated from these response surface at different level of $\alpha$-cuts. In another sense, statistical properties could be generated at different level of possibility.

\section{References}

Abrahamsson M M 2002 Uncertainty in quantitative risk analysis-Characterization and methods of treatment. Report 1024 Lund

Adomian G 1980 Applied stochastic processes, New York. Academic press. pp. 1-17

Baudrit C, Guyonnet D and Dubois D 2007 Joint propagation of variability and imprecision in assessing the risk of groundwater contamination. J. Contaminant Hydrology 93: 72-84

Brandimarte P 2011 Quantitative methods: An introduction for business management. New Jersey: John Wiley \& Sons

Box G E P and Draper N R 1987 Empirical model-building and response surfaces. New York: John Wiley $\&$ Sons

Box G E P, Hunter W G and Hunter J S 1978 Statistics for experimenters: An introduction to design, data analysis and model building. New York: John Wiley \& Sons 
Chen Z, Zhao L and Lee K 2010 Environmental risk assessment of offshore produced water discharges using a hybrid fuzzy-stochastic modeling approach. Environmental Modelling and Software 25: 782-792

Chutia R, Mahanta S and Datta D 2013 Sensitivity analysis of atmospheric dispersion model-RIMPUFF using the hartley-like measure. J. Appl. Mathematics and Informatics 31(1-2): 99-110

Chutia R, Mahanta S and Datta D 2013 Non-probabilistic sensitivity and uncertainty analysis of atmospheric dispersion. Annals of Fuzzy Mathematics and Informatics 5(1): 213-22

Chutia R, Mahanta S and Datta D 2013 Uncertainty modelling of atmospheric dispersion model using fuzzy set and imprecise probability. J. Intelligent and Fuzzy Systems 25: 737-746

Colvile R N, Woodfield N K, Carruthers D J, Fisher B E A, Rickard A, Neville S and Hughes A 2002 Uncertainty in dispersion modelling and urban air quality mapping. J. Environmental Sci. Policy 5: 202-220

Datta D and Kushwaha H S 2011 Uncertainty quantification using stochastic response surface method case study-transport of chemical contaminants through groundwater. Int. J. Energy Information and Commun. 2(3): 49-58

Du X and Chen W 2001 A most probable point based method for uncertainty analysis. J. Design and Manufacturing Automation 4(1): 47-66

Dubois D and Parde H 1988 Possibility theory: An approach to computerized processing of uncertainty. New York: Plenum Press

Eimutis E C and Konicek M G 1972 Derivations of continuous functions for the lateral and vertical atmospheric dispersion coefficients. Atmospheric Environment 16: 859-863

Fakhraee H, Saeedi M and Rezaei Sadrabadi M 2007 A fuzzy air pollution dispersion model. In Proceeding ASM '07 The 16th IASTED International Conference on Applied Simulation and Modelling

Faybishenko B 2010 Fuzzy-probabilistic calculations of water-balance uncertainty. Stochastic Environmental Research and Risk Assessment 24: 939-952

Ferson S, Root W and Kuhn R 1999 Ramas Risk Calc: Risk assessment with uncertain numbers. New York, Setauket: Lewis Publisher

Ferson S and Ginzburg L 1995 Hybrid arithmetic. In: Proceedings of the 1995 joint ISUMA/NAFIPS conference. IEEE Computer Society Press, Los Alamitos, California, pp. 619-623

Frey H C and Bammi S 2003 Probabilistic nonroad mobile source emission factors. J. Env. Eng. 129(2): $162-168$

Guyonnet D, Bourgine B, Dubois D, Fargier H, Come B and Chiles J 2003 A hybrid approach for addressing uncertainty in risk assessments. J. Env. Eng. 126: 68-78

Heng L L, Huang G H and Zou Y 2008 An integrated fuzzy-stochastic modelling approach for assessing health-impact risk from air pollution. Stochastic Environmental Research and Risk Assessment 22: 789-803

Hessian W C, Strom D E and Haan C T 1996 Two-phase uncertainty analysis: An example using the universal soil loss equation. Transactions of The ASAE 39(4): 1309-1319

Hoybe J A 1998 Error propagation and data collection design. An application in water quality modeling. Water, Air and Soil Pollution 103(1-4): 101-119

Ibrahim R A 1992 Structural dyanimics with parameters uncertainties. Appl. Mechanics Reviews 40(3): 309-328

Isukapalli S S 1999 Uncertainty analysis of transport-transformation models. PhD Dissertation, Rutgers University

Isukapalli S S and Georgopoulos P G 1998 Stochastic response surface methods (SRSMs) for uncertainty propagation: Application to environmental and biological systems. Risk Analysis 18(3): 351-363

Kentel E and Aral M M 2004 Probabilistic-fuzzy health risk modelling. Stochastic Environmental Research and Risk Assessment 18: 324-338

Kentel E and Aral M M 2005 2D Monte Carlo versus 2D Fuzzy Monte Carlo health risk assessment. Stochastic Environmental Research and Risk Assessment 19: 86-96

Mahadevan S and Raghothamachar P 2000 Adaptive simulation for system reliability analysis of large structures. Computers and Structures 77: 725-734 
Nguyen H T, Kreinovich V, Wu B and Xiang G 2012 Computing statistics under interval and fuzzy uncertainty. Berlin, Heidelberg: Springer-Verlag

Qin X S 2012 Assessing environmental risks through fuzzy parametrized probabilistic analysis. Stochastic Environmental Research and Risk Assessment 26: 43-58

Saeedi M, Fakhraee H and Rezaei Sadrabadi M 2008 A fuzzy modified gaussian air pollution dispersion model. Research J. Env. Sci. 2(3): 156-169

Sengupta A and Pal T K 2000 On comparing interval numbers. European J. Operational Research 127: $28-43$

Tucker W T and Ferson S 2003 Probability bounds analysis in environmental risk assessment. Setauket, New York: Appl. Biomathematics

Wang C 1999 Parametric uncertainty analysis for complex engineering systems. Ph. D. Thesis MIT 53-113

Wiener S 1938 The homogeneous chaos. American J. Mathematics 60: 897-936

Yang A L, Huang G H and Qin X S 2010 An integrated simulation-assessment approach for evaluating health risks of groundwater contamination under multiple uncertainties. Water Resource and Management 24: 3349-3369

Zadeh L A 1965 Fuzzy sets. Information Control 8: 338-353

Zadeh L A 1978 Fuzzy sets as a basis for theory of possibility. Fuzzy Sets and System 1(1): 3-28 